\title{
Infected urachal cyst in a young adult: A case report
}

\author{
Amar Varshney ${ }^{1}$, Rajesh Khanna ${ }^{2}$, Arvind Jain ${ }^{3}$ \\ From ${ }^{1}$ Wing Commander/Classified Specialist Surgery, Department of Surgery, 9 Air Force Hospital, ${ }^{2}$ Lieutenant Colonel/Surgeon, Department of \\ Surgery, Military Hospital Jalandhar Cantt, Halwara, Punjab, ${ }^{3}$ Colonel/Senior Advisor Surgery, Department of Surgery, Base Hospital Delhi Cantt, \\ New Delhi, India
}

\begin{abstract}
The urachal cyst is one of a spectrum of urachal abnormalities most commonly found in children. They are very rarely seen in adults because the urachus is normally obliterated in early infancy. Clinical presentation is non-specific; therefore, a high index of suspicion is required to make the diagnosis. When it is diagnosed, surgical excision is advised due to the risk of malignant transformation. We describe the case of a 26-year-old male who presented with a small swelling of the umbilicus and purulent umbilical discharge. The diagnosis of an infected urachal cyst was confirmed on ultrasonography abdomen and contrast-enhanced computed tomography abdomen. He was treated initially with broad-spectrum antibiotics and then by surgical excision of the cyst and fibrous tract. Histology of the excised specimen showed chronic inflammation with no evidence of malignancy. Post-operative recovery was uneventful.
\end{abstract}

Key words: Abscess, Urachal cyst, Urachal remnant, Urachal sinus, Urachus

$\mathrm{T}$ he urachus is the remnant of the cloaca, which in adults attaches the bladder dome to the umbilicus. After birth, it obliterates and presents as the midline umbilical ligament [1]. Patent urachal anomalies are usually detected in childhood. In adults, they occur very rarely and the presentation and diagnosis may be occasionally challenging [2]. It is important to remember the possibility of infected urachal remnants in a patient presenting with an acute abdomen in the emergency department. When it is diagnosed, surgical excision is advised due to the risk of malignant transformation [3].

\section{CASE REPORT}

A 26-year-old man presented to our center with a complaint of 1 week of abdominal pain. The character of his pain was moderate cramping with nausea and vomiting. The vomiting was nonprojectile, had multiple episodes of vomiting, and contained undigested food particles. It was associated with low-grade fever but without lower urinary tract symptoms. He never experienced spontaneous extrusion of fluid from his umbilicus.

On physical examination, he looked well with a pulse rate of $82 / \mathrm{min}$ and blood pressure of $124 / 82 \mathrm{~mm}$ of $\mathrm{Hg}$, and his body temperature was $38.5^{\circ} \mathrm{C}$. The abdomen was soft with small swelling of the umbilicus and tenderness was present over the

\section{Access this article online}

Received - 25 August 2020

Initial Review - 08 September 2020

Accepted - 03 October 2020

DOI: $10.32677 /$ IJCR.2020.v06.i10.007 swelling (Fig. 1). No distension or peritoneal signs were elicited, and his rectal examination was normal. There was a minimal amount of seropurulent discharge from the umbilicus.

Laboratory data revealed a white blood cell count of 11,000/L. Blood biochemistry and urine analysis were normal. His X-ray abdomen was normal. Abdominal ultrasonography (USG) revealed a tract from the umbilicus to the abdominal wall and a hypoechoic mass with hetero echogenic content between the peritoneum, the muscle layer, and the bladder, without the presence of fluid in the peritoneal cavity. The suggested diagnosis was the infection of the urachal remnants.

The treatment was initiated with broad empirical (Inj amoxiclav $1.2 \mathrm{~g}$ I.V. BD and Inj gentamicin $80 \mathrm{mg}$ I.V. BD) antibiotics. However, his condition did not fully recover, so he underwent contrast-enhanced computed tomography (CT) abdomen which confirmed the same diagnosis.

The patient was taken up for laparotomy with a midline incision (Fig. 2a) and the pus was drained (Fig. 2b). The extraperitoneal excision of the urachal remnant with the cuff of the urinary bladder was performed (Fig. 3). There were no postoperative complications.

On pathological examination, the surgical tissue was mainly composed of fatty and dense fibrous tissues without any cell tumor of the bladder wall. The result of a pus culture showed an Escherichia coli infection which was treated with the antibiotic ciprofloxacin. At his follow-up examination 6 months later, he was asymptomatic and had no abnormalities of the abdominal wall.

Correspondence to: Amar Varshney, Department of Surgery, 9 Air Force Hospital, Ludhiana - 141 106, Punjab, India. E-mail: amarvarshney@yahoo.co.in

(C) 2020 Creative Commons Attribution-NonCommercial 4.0 International License (CC BY-NC-ND 4.0). 


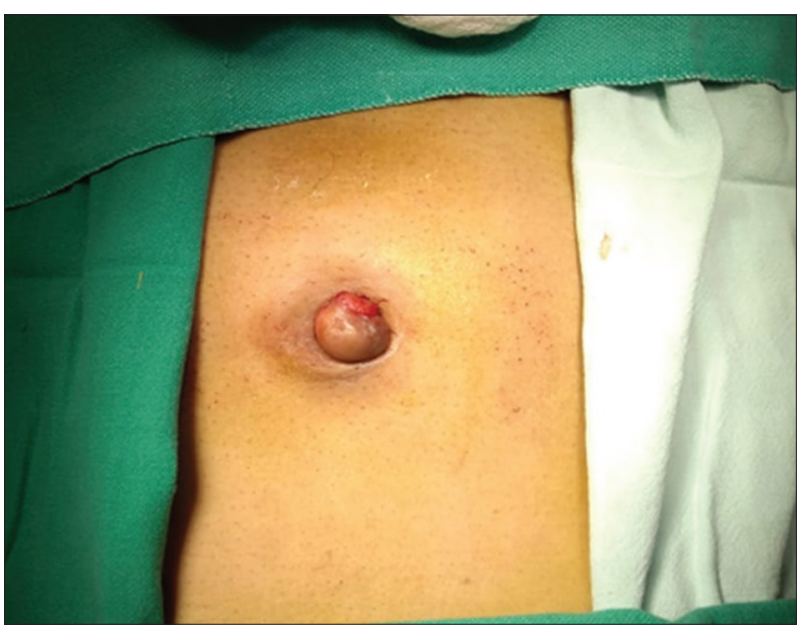

Figure 1: The patient with swelling at umbilicus
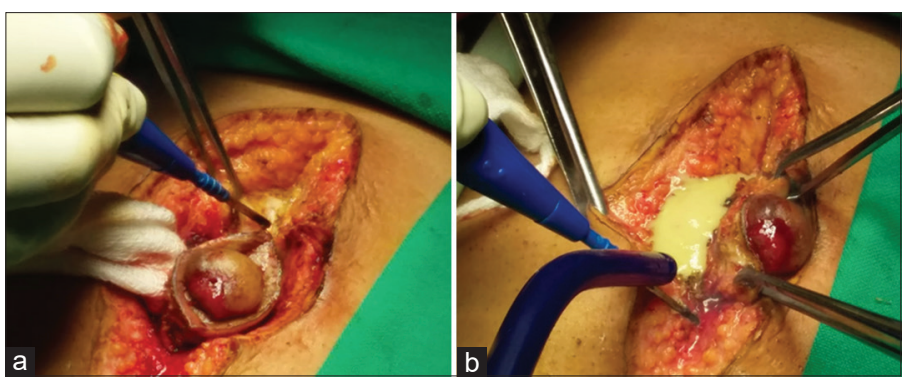

Figure 2: (a) Midline laparotomy incision with excision of the umbilicus; (b) drainage of abscess

\section{DISCUSSION}

The urachus is a normal embryonic remnant of the primitive bladder dome. It generally exists as a fibrous cord extending from the dome of the bladder to the umbilicus. It also occupies the potential midline space between the peritoneum and fascia transversalis. Urachal diseases can be congenital or acquired [4].

Congenital anomalies occur when the urachus fails to obliterate. The pathology associated with congenital disorder urachus is generally divided into four categories. The first is a patent urachus, in which there is a communication between the bladder and the umbilicus. Second is the umbilical sinus, in which the urachus opens into the umbilicus. Here, drainage from the umbilicus will often be present. The third category is the vesicourachal diverticulum, in which the urachus has a wide patent opening into the bladder. Urinary complaints are often present with this type. The last type is the urachal cyst, in which the urachus has a cyst-like structure within its length. The urachal cyst becomes prominent when infection occurs or ruptures of the cyst [5].

Acquired diseases include inflammation and neoplasm. Inflammation occurs more frequently in children and young adults. Infected urachal cysts with an onset beyond the fifth decade are quite rare. The urachus is located in a clinically silent area, extraperitoneally in the space of Retzius. As a consequence, possible symptoms and clinical signs of inflammation as well as of tumors are in many cases non-specific or delayed, or even absent. Inflammation, as well as the development of an abscess,

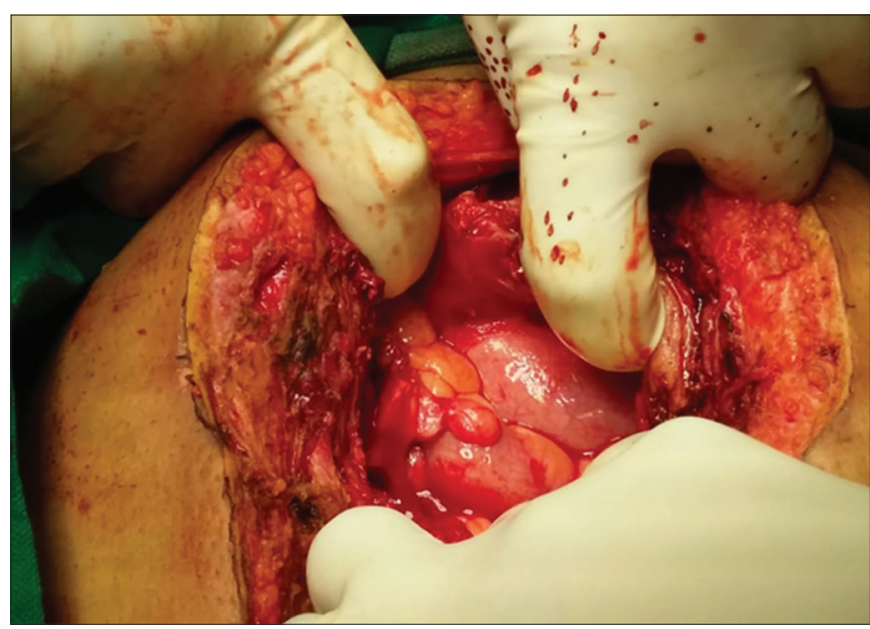

Figure 3: Excision of whole tract till the urinary bladder

can remain clinically unrecognized for a long time or it can be considered as an acute surgical abdomen.

USG is usually sufficient to diagnose an infected urachal remnant. Abscesses may have a solid appearance on USG [3] as well as attenuation values higher than water on CT, thus mimicking a neoplasm. On the other hand, tumors may show on CT hypodense central areas due to necrosis, hemorrhage, or mucoid content such that the resulting features may simulate an inflammatory mass [6].

The most common infecting pathogens are E. coli and Proteus, but a variety of other pathogens can also be found [7]. The differential diagnosis of urachal abscess should include cellulitis, necrotizing fasciitis, peritonitis, acute appendicitis, hematoma, ventral or umbilical hernia, and tumor lesions, especially when it develops into the abdominal wall [8].

The treatment of infectious urachal remnants in adulthood should include antibiotic therapy for the acute infection followed by primary or secondary excision after draining the abscess cavity. It is generally recommended that all urachal remnants should be excised to avoid recurrent disease due to possible malignant transformation later in life. However, when the infection is present, management by pre-operative percutaneous drainage and subsequent elective excision may represent the most effective surgical option. Although the condition is not well defined, the possibility of adenocarcinoma in an incompletely resected specimen led to the practice of radical excision of the urachal remnant. Radical excision requires removing all the structures within the umbilicovesical fascia [9], including the urachus and each medial umbilical ligament, as well as the associated peritoneum from the umbilicus to the bladder dome. For benign lesions that do not communicate with the umbilicus or bladder, there is no consensus on whether the umbilicus and a bladder cuff should be resected routinely [10] Most reports of urachal cyst excision do not mention umbilical resection. Traditional surgical excision of urachal remnant involves a transverse or midline incision.

\section{CONCLUSION}

Urachal abnormalities are rare in adults. Clinical presentation is non-specific; therefore, a high index of suspicion is required to 
make the diagnosis. When it is diagnosed, surgical excision is advised due to the risk of malignant transformation.

\section{REFERENCES}

1. Tazi F, Ahsaini M, Khalouk A, Mellas S, Stuurman-Wieringa RE, Elfassi MJ, et al. Abscess of urachal remnants presenting with acute abdomen: A case series. J Med Case Rep 2012;6:226.

2. Ash A, Gujral R, Raio C. Infected urachal cyst initially misdiagnosed as an incarcerated umbilical hernia. J Emerg Med 2012;42:171-3.

3. Ueno T, Hashimoto H, Yokoyama H, Ito M, Kouda K, Kanamaru H. Urachal anomalies: Ultrasonography and management. J Pediatr Surg 2003;38:1203-7.

4. Walker C. A case report of urachal abscess: A rare differential in adult abdominal pain. Hawaii Med J 2010;69:35-6.

5. Hsu CC, Liu YP, Lien WC, Lai TI, Chen WJ, Wang HP. Urachal abscess: A cause of adult abdominal pain that cannot be ignored. Am J Emerg Med 2005;23:229-30.

6. Yu JS, Kim KW, Lee HJ, Lee YJ, Yoon CS, Kim MJ. Urachal remnant diseases: Spectrum of CT and US findings. Radiographics 2001;21:451-61.

7. MacNeily AE, Koleilat N, Kiruluta HG, Homsy YL. Urachal abscesses: Protean manifestations, their recognition, and management. Urology 1992;40:530-5

8. Villavicencio CP, Adam SZ, Nikolaidis P. Imaging of the urachus: Anomalies, complications, and mimics. Radiographics 2016;36:2049-63.

9. Copp HL, Wong IY, Krishnan C, Malhotra S, Kennedy WA. Clinical presentation and urachal remnant pathology: Implications for treatment. J Urol 2009;182:1921-4.

10. Hassanbhai DH, Ng FC, Koh LT. Is excision necessary in the management of adult urachal remnants?: A 12-year experience at a single institution. Scand J Urol 2018;52:432-6.

Funding: None; Conflicts of Interest: None Stated.

How to cite this article: Varshney A, Khanna R, Jain A. Infected urachal cyst in a young adult: A case report. Indian J Case Reports. 2020;6(10):566-568. 